\title{
SYMMETRIES OF A GENERIC COACTION
}

\author{
TEODOR BANICA
}

\begin{abstract}
If $B$ is $\mathbf{C}^{*}$-algebra of dimension $4 \leq n<\infty$ then the finite dimensional irreducible representations of the compact quantum automorphism group of $B$, say $G_{\text {aut }}(\widehat{B})$, have the same fusion rules as the ones of $\mathbf{S O}(3)$. As consequences, we get (1) a structure result for $G_{\text {aut }}(\widehat{B})$ in the case where $B$ is a matrix algebra (2) if $n \geq 5$ then the dual $\widehat{G}_{\text {aut }}(\widehat{B})$ is not amenable (3) if $n \geq 4$ then the fixed point subfactor $P^{G_{a u t}(\widehat{B})} \subset(B \otimes P)^{G_{a u t}(\widehat{B})}$ has index $n$ and principal graph $A_{\infty}$.
\end{abstract}

\section{INTRODUCTION}

Let $X_{n}$ be the space consisting of $n$ points. The category of groups acting on $X_{n}$ has a universal object: the symmetric group $\mathcal{S}_{n}$. S. Wang has recently discovered that when replacing "groups" with "compact quantum groups" the resulting category has also a universal object, say $G_{\text {aut }}\left(X_{n}\right)$. If $n=1,2,3$ then $G_{a u t}\left(X_{n}\right)=\mathcal{S}_{n}$. If $n \geq 4$ this compact quantum group $G_{a u t}\left(X_{n}\right)$ is not a classical group, nor a finite quantum group (see [9], [10]) and very less seems to be known about it. Some quantum subgroups of it, which are of interest in connection with spin models, were constructed in [0] ].

More generally, any finite dimensional $\mathbf{C}^{*}$-algebra $B$ has a compact quantum group of automorphisms, say $G_{\text {aut }}(\widehat{B})$ (we have $G_{\text {aut }}\left(\widehat{\mathbf{C}}^{n}\right)=G_{\text {aut }}\left(X_{n}\right)$ ). See [9]. See also theorem 1.1 below and the comments preceding it: actually when $B$ is noncommutative the definition of $G_{\text {aut }}(\widehat{B})$ requires as data a trace on $B$-i.e. one can define only compact quantum groups of automorphisms of measured quantum finite spaces, cf. [9] - and the distinguished trace we use here is in general different from the distinguished trace used in [9].

In this paper we prove that if $\operatorname{dim}(B) \geq 4$ then the set of classes of finite dimensional irreducible representations of $G_{a u t}(\widehat{B})$ can be labeled by the positive integers, $\operatorname{Irr}\left(G_{\text {aut }}(\widehat{B})\right)=\left\{p_{n} \mid n \in \mathbf{N}\right\}$, such that the fusion rules are

$$
p_{k} \otimes p_{s}=p_{|k-s|}+p_{|k-s|+1}+\cdots+p_{k+s-1}+p_{k+s}
$$

In other words, we prove that we have an isomorphism of fusion semirings

$$
R^{+}\left(G_{\text {aut }}(\widehat{B})\right) \simeq R^{+}(\mathbf{S O}(3))
$$

This kind of isomorphisms already appeared in quite various contexts, and seem to be deeply related to notions of "deformation". See the survey [4].

The reasons for the existence of the above isomorphism are somehow hidden by the technical details of the proof, and will be explained now.

"4" comes from Jones' index (cf. proposition 2.2); and also from Wang's computations for $n=1,2,3$. The fact that the distinguished trace on $B$ we use is the "good"

e-mail address: banica@math.jussieu.fr. 
one comes from Markov inclusions (cf. proposition 2.1 (ii)); and also from our result itself. For other traces proposition 2.1 (iv) shows that the fundamental representation of the corresponding $G_{\text {aut }}(\widehat{B})$ contains, besides the trivial representation, at least two components. This situation reminds the one of $A_{o}(F)$ 's for $F \bar{F} \notin \mathbf{C} \cdot I d$ (see [11] for what happens in this case).

The category $\operatorname{Rep}\left(G_{\text {aut }}(\widehat{B})\right)$ of representations of $G_{\text {aut }}(\widehat{B})$ is in a certain sense "generated" by two arrows: the multiplication $\mu: B \otimes B \rightarrow B$ and the unit $\eta: \mathbf{C} \rightarrow B$ (see proposition 1.1). The point is that the only "relations" satisfied by these "generators" are the "universal" ones coming from the axioms of the algebra structure of $B$ (see lemma 2.1). This shows that $\operatorname{Rep}\left(G_{\text {aut }}(\widehat{B})\right)$ "does not depend so much on $B$ ". More precisely, with a good index and a good trace, one can show that its semiring of objects - which is $R^{+}\left(G_{\text {aut }}(\widehat{B})\right)$ - does not depend on $B$. On the other hand one can see from definitions (see also corollary 4.1) that for $B=M_{2}(\mathbf{C})$ we have

$$
\left.G_{\text {aut }}\left(\widehat{M_{2}(\mathbf{C}}\right)\right) \simeq \mathbf{P U}(2) \simeq \mathbf{S O}(3)
$$

with $\mathbf{P U}(2)$ acting on $M_{2}(\mathbf{C})$ in the obvious way. Thus $R^{+}\left(G_{\text {aut }}(\widehat{B})\right) \simeq R^{+}(\mathbf{S O}(3))$.

The paper is organized as follows. In section 1 we find convenient "presentations" of the Hopf $\mathbf{C}^{*}$-algebra of continuous functions on $G_{a u t}(\widehat{B})$ and of its category of corepresentations. In section 2 we use theory from [6], [5] for finding the good index and the good trace on $B$. In sections 3 and 4 we use techniques from [1] for proving the main result. By combining it with results from [1], [2], [3] we get the consequences (1), (2), (3) listed in the abstract.

\section{Coactions And Corepresentations}

There is only one Hopf $\mathbf{C}^{*}$-algebra to be considered in this paper, namely $A^{\text {aut }}(B)$, the object dual to the compact quantum group $G_{a u t}(\widehat{B})$ in the introduction. $A^{\text {aut }}(B)$ is by definition a certain $\mathbf{C}^{*}$-algebra given with generators and relations. Its precise structure is that of a finitely generated full Woronowicz-Kac algebra.

However, for understanding the definition of $A^{\text {aut }}(B)$ we have to state one of its universality properties. The really relevant one holds in the category of finitely generated full Woronowicz-Kac algebras.

The definition of these algebras is as follows (see [4] for explanations on terminology).

Definition 1.1 (cf. definition 1.1 in [12]). A finitely generated (or co-matricial) full Woronowicz-Kac algebra is a pair $(A, u)$ consisting of a unital $\mathbf{C}^{*}$-algebra $A$ and a unitary matrix $u \in M_{n}(A)$ satisfying the following conditions:

(i) $A$ is the enveloping $\mathbf{C}^{*}$-algebra of its $*$-subalgebra $\mathcal{A}$ generated by the entries of $u$.

(ii) there exists a $\mathbf{C}^{*}$-morphism $\Delta: A \rightarrow A \otimes A$ such that $(i d \otimes \Delta) u=u_{12} u_{13}$.

(iii) there exists a $\mathbf{C}^{*}$-antimorphism $S: A \rightarrow A$ sending $u_{i j} \leftrightarrow u_{j i}^{*}$.

One can define a $\mathbf{C}^{*}$-morphism $\varepsilon: A \rightarrow \mathbf{C}$ by $\varepsilon\left(u_{i j}\right)=\delta_{i, j}$. The maps $\Delta, \varepsilon, S$ satisfy the well-known requirements for a comultiplication, counit and antipode. More precisely, their restrictions to $\mathcal{A}$ make $\mathcal{A}$ a Hopf $\mathrm{C}$-algebra. See [12].

Let $V$ be a finite dimensional $\mathbf{C}$-linear space. A coaction of the Hopf algebra $\mathcal{A}$ on $V$ is a linear map $\beta: V \rightarrow V \otimes \mathcal{A}$ satisfying

$$
(i d \otimes \Delta) \beta=(\beta \otimes i d) \beta, \quad(i d \otimes \varepsilon) \beta=i d
$$


A corepresentation of $\mathcal{A}$ on $V$ is an element $u \in \mathcal{L}(V) \otimes \mathcal{A}$ satisfying

$$
(i d \otimes \Delta) u=u_{12} u_{13}, \quad(i d \otimes \varepsilon) u=1
$$

The coactions (resp. corepresentations) of the Hopf algebra $\mathcal{A}$ will be called coactions (resp. corepresentations) of the finitely generated full Woronowicz-Kac algebra $A$. These two notions are related as follows.

Lemma 1.1. Denote by $\beta \mapsto u_{\beta}$ the composition of canonical linear isomorphisms

$$
\mathcal{L}(V, V \otimes \mathcal{A}) \simeq V^{*} \otimes V \otimes \mathcal{A} \simeq \mathcal{L}(V) \otimes \mathcal{A}
$$

and by $u \mapsto \beta_{u}$ its inverse. That is, if $\left\{v_{i}\right\}$ is a basis of $V$ and $\left\{e_{i j}: v_{j} \mapsto v_{i}\right\}$ is the corresponding system of matrix units in $\mathcal{L}(V)$, then the relation between $\beta$ and $u_{\beta}$ is

$$
\beta\left(v_{i}\right)=\sum_{j} v_{j} \otimes u_{j i} \Longrightarrow u_{\beta}=\sum_{i j} e_{i j} \otimes u_{i j}
$$

If $\beta: V \rightarrow V \otimes \mathcal{A}$ is a linear map, then $\beta$ is a coaction iff $u_{\beta}$ is a corepresentation.

Let $B$ be a finite dimensional $\mathbf{C}^{*}$-algebra. We denote by $\mu: B \otimes B \rightarrow B$ the multiplication and by $\eta: \mathbf{C} \rightarrow B$ the linear map which sends $1 \mapsto 1$. Let $\operatorname{tr}: B \rightarrow \mathbf{C}$ be a faithful positive normalised trace (in what follows we call such traces just "traces"). We recall that the scalar product $\langle x, y\rangle=\operatorname{tr}\left(y^{*} x\right)$ makes $B$ into a Hilbert space.

Let $A$ be a finitely generated full Woronowicz-Kac algebra. A coaction of $A$ on $(B, t r)$ is a morphism of unital $*$-algebras $\beta: B \rightarrow B \otimes \mathcal{A}$ which is a coaction of $A$ on the $\mathbf{C}$-linear space $B$, and which is such that the trace satisfies the equivariance property

$$
(i d \otimes \operatorname{tr}) \beta=\operatorname{tr}(.) 1
$$

We will use the following notations. If $D$ is a unital $\mathbf{C}$-algebra and $V, W$ are two finite dimensional C-linear spaces and $v \in \mathcal{L}(V) \otimes D$ and $w \in \mathcal{L}(W) \otimes D$ we define

$$
\begin{gathered}
v \otimes w=v_{13} w_{23} \in \mathcal{L}(V) \otimes \mathcal{L}(W) \otimes D \\
H o m(v, w)=\{T \in \mathcal{L}(V, W) \mid(T \otimes i d) v=w(T \otimes i d)\}
\end{gathered}
$$

If $D$ is a bialgebra and $v$ and $w$ are corepresentations, then $\otimes$ and Hom are the usual tensor product and space of intertwiners. In general, it is possible to associate to any algebra $D$ a certain monoidal category having these $H o m$ and $\otimes$, so our notations are not as abusive as they seem.

Lemma 1.2. Let $u \in \mathcal{L}(B) \otimes \mathcal{A}$ be a corepresentation, and denote $\beta:=\beta_{u}$.

(i) $\beta$ is multiplicative $\Longleftrightarrow \mu \in \operatorname{Hom}\left(u^{\otimes 2}, u\right)$.

(ii) $\beta$ is unital $\Longleftrightarrow \eta \in \operatorname{Hom}(1, u)$.

(iii) $\operatorname{tr}$ is $\beta$-equivariant $\Longleftrightarrow \eta \in \operatorname{Hom}\left(1, u^{*}\right)$.

If these conditions are satisfied, then:

(iv) $\beta$ is involutive $\Longleftrightarrow u$ is unitary.

Proof. Let $\left\{b_{1}, b_{2}, \ldots, b_{n}\right\}$ be an orthonormal basis of the Hilbert space $B$. We will use many times the formula

$$
x=\sum_{i} \operatorname{tr}\left(b_{i}^{*} x\right) b_{i}=\sum_{i} \operatorname{tr}\left(x b_{i}^{*}\right) b_{i}=\sum_{i} \operatorname{tr}\left(b_{i} x\right) b_{i}^{*}=\sum_{i} \operatorname{tr}\left(x b_{i}\right) b_{i}^{*}
$$


for any $x \in B$, and especially its particular case $1=\sum \operatorname{tr}\left(b_{i}^{*}\right) b_{i}=\sum \operatorname{tr}\left(b_{i}\right) b_{i}^{*}$. For any $i, j$ we have

$$
\begin{gathered}
(\mu \otimes 1) u^{\otimes 2}\left(b_{i} \otimes b_{j} \otimes 1\right)=(\mu \otimes 1)\left(\sum_{k l} b_{k} \otimes b_{l} \otimes u_{k i} u_{l j}\right)=\sum_{k l} b_{k} b_{l} \otimes u_{k i} u_{l j}=\beta\left(b_{i}\right) \beta\left(b_{j}\right) \\
u(\mu \otimes 1)\left(b_{i} \otimes b_{j} \otimes 1\right)=u\left(\sum_{k} b_{k} \otimes \operatorname{tr}\left(b_{i} b_{j} b_{k}^{*}\right) 1\right)=\sum_{k l} b_{l} \otimes \operatorname{tr}\left(b_{i} b_{j} b_{k}^{*}\right) u_{l k}=\beta\left(b_{i} b_{j}\right)
\end{gathered}
$$

and (i) follows. The assertion (ii) is clear from

$$
u(1 \otimes 1)=u\left(\sum_{j} b_{j} \otimes \operatorname{tr}\left(b_{j}^{*}\right) 1\right)=\sum_{i j} b_{i} \otimes \operatorname{tr}\left(b_{j}^{*}\right) u_{i j}=\sum_{j} \operatorname{tr}\left(b_{j}^{*}\right) \beta\left(b_{j}\right)=\beta(1)
$$

As for (iii), we have $1 \otimes 1=\sum_{j} b_{j} \otimes\left(\operatorname{tr}\left(b_{j}\right) 1\right)^{*}$ and

$u^{*}(1 \otimes 1)=\left(\sum_{i j} e_{j i} \otimes u_{i j}^{*}\right)\left(\sum_{I} b_{I} \otimes \operatorname{tr}\left(b_{I}^{*}\right) 1\right)=\sum_{i j} b_{j} \otimes \operatorname{tr}\left(b_{i}^{*}\right) u_{i j}^{*}=\sum_{j} b_{j} \otimes\left((\operatorname{tr} \otimes i d) \beta\left(b_{j}\right)\right)^{*}$

Let us prove (iv). Assume that (i,ii,iii) are satisfied and that $\beta$ is involutive. Then for any $i, k$ we have

$$
\sum_{j} u_{j i}^{*} u_{j k}=(\operatorname{tr} \otimes i d)\left(\sum_{j s} b_{j}^{*} b_{s} \otimes u_{j i}^{*} u_{s k}\right)=(\operatorname{tr} \otimes i d) \beta\left(b_{i}^{*} b_{k}\right)=\operatorname{tr}\left(b_{i}^{*} b_{k}\right) 1=\delta_{i, k}
$$

so $u^{*} u=1$. On the other hand, as $u$ is a corepresentation of the Hopf algebra $\mathcal{A}$, we know that $u$ is invertible (its inverse is $(i d \otimes S) u$ : this follows by considering $(i d \otimes E) u$, with $E=m(S \otimes i d) \Delta=m(i d \otimes S) \Delta=\varepsilon() 1$.$) . Thus u$ is a unitary. Conversely, assume that (i,ii,iii) are satisfied and that $u$ is unitary. From $\mu \in \operatorname{Hom}\left(u^{\otimes 2}, u\right)$ we get $\mu^{*} \in \operatorname{Hom}\left(u, u^{\otimes 2}\right)$, and together with $\eta \in \operatorname{Hom}(1, u)$ this gives $\mu^{*} \eta \in \operatorname{Hom}\left(1, u^{\otimes 2}\right)$. As $u^{\otimes 2}=u_{13} u_{23}$ it follows that

$$
u_{23}\left(\mu^{*} \eta(1) \otimes 1\right)=u_{13}^{*}\left(\mu^{*} \eta(1) \otimes 1\right)
$$

We have $<\mu^{*} \eta(1), b_{p} \otimes b_{q}>=<1, b_{p} b_{q}>=\operatorname{tr}\left(b_{p}^{*} b_{q}^{*}\right)$ for any $p, q$, so that

$$
\mu^{*} \eta(1)=\sum_{p q} \operatorname{tr}\left(b_{p}^{*} b_{q}^{*}\right) b_{p} \otimes b_{q}
$$

Let us compute $u_{23}\left(\mu^{*} \eta(1) \otimes 1\right)$ and $u_{13}^{*}\left(\mu^{*} \eta(1) \otimes 1\right)$ by using this formula:

$$
\begin{aligned}
& u_{23}\left(\mu^{*} \eta(1) \otimes 1\right)=\left(\sum_{i s} i d \otimes e_{i s} \otimes u_{i s}\right)\left(\sum_{j S} \operatorname{tr}\left(b_{j}^{*} b_{S}^{*}\right) b_{j} \otimes b_{S} \otimes 1\right)=\sum_{j i s} b_{j} \otimes b_{i} \otimes \operatorname{tr}\left(b_{j}^{*} b_{s}^{*}\right) u_{i s} \\
& u_{13}^{*}\left(\mu^{*} \eta(1) \otimes 1\right)=\left(\sum_{j k} e_{j k} \otimes i d \otimes u_{k j}^{*}\right)\left(\sum_{i K} \operatorname{tr}\left(b_{K}^{*} b_{i}^{*}\right) b_{K} \otimes b_{i} \otimes 1\right)=\sum_{j i k} b_{j} \otimes b_{i} \otimes \operatorname{tr}\left(b_{k}^{*} b_{i}^{*}\right) u_{k j}^{*}
\end{aligned}
$$

Thus from $u_{23}\left(\mu^{*} \eta(1) \otimes 1\right)=u_{13}^{*}\left(\mu^{*} \eta(1) \otimes 1\right)$ we get that

$$
\sum_{s} \operatorname{tr}\left(b_{j}^{*} b_{s}^{*}\right) u_{i s}=\sum_{k} \operatorname{tr}\left(b_{k}^{*} b_{i}^{*}\right) u_{k j}^{*}
$$

for any $i$. The fact that $\beta$ is involutive follows from this and from

$$
\beta\left(b_{j}^{*}\right)=\sum_{s} \beta\left(b_{s}\right) \operatorname{tr}\left(b_{j}^{*} b_{s}^{*}\right)=\sum_{i s} b_{i} \otimes \operatorname{tr}\left(b_{j}^{*} b_{s}^{*}\right) u_{i s}
$$




$$
\beta\left(b_{j}\right)^{*}=\sum_{k} b_{k}^{*} \otimes u_{k j}^{*}=\sum_{i k} b_{i} \otimes \operatorname{tr}\left(b_{k}^{*} b_{i}^{*}\right) u_{k j}^{*}
$$

We will use now lemmas 1.1 and 1.2 for associating to any pair $(B, t r)$ a certain algebra $A^{\text {aut }}(B, t r)$ and a category $\mathcal{C}_{B, t r}$. The theorem 1.1 below claims no originality and may be found, in a slightly different form, in [9]. Notice the following two differences between it and theorem 5.1 in [9].

(1) Only the case of a certain distinguished trace was explicitely worked out in 99, and the general case was left in there to the reader. What happens is that Wang's trace is not the "good" one, i.e. it is in general different from the one needed for having "minimality" of $R^{+}\left(A^{\text {aut }}(B, t r)\right)$ (see the comments in the introduction), which is called "canonical trace" in section 2 below. By the way, this is the reason why we will use later on the notation $A^{\text {aut }}(B)$ (for $A^{\text {aut }}(B, t r)$ with $t r=$ the canonical trace in the sense of definition 2.1 below) instead of Wang's notation $A_{\text {aut }}(B)$ (which corresponds to $A^{\text {aut }}(B, t r)$ with $t r=$ the distinguished trace used in [9]): these two algebras may not be isomorphic in general.

(2) The presentation of $A^{\text {aut }}(B, t r)$ given here - to be used in proposition 1.1 for finding a "presentation" of its category of corepresentations - is different from the one in [9]. The point is that this kind of presentation is the "good" one in a certain (quite obvious) sense (see section 2 in 4 (1). Of course one can prove, via manipulations of generators and relations, or just by using uniqueness of universal objects, that our algebra $A^{\text {aut }}(B, t r)$ is the same as Wang's $A_{a u t}(B, t r)$. For reasons of putting aut as an exponent in our notation see the above comment (1).

Theorem 1.1 (cf. [9]). Let $(B, t r)$ be a finite dimensional $\mathbf{C}^{*}$-algebra together with a trace. Denote by $\mu: B \otimes B \rightarrow B$ the multiplication and by $\eta: \mathbf{C} \rightarrow B$ the linear unital map. Choose an orthonormal basis of $B$, and use it for identifying $B \simeq \mathbf{C}^{n}$ as Hilbert spaces, with $n=\operatorname{dim}(B)$. Consider the following universal $\mathbf{C}^{*}$-algebra $A^{\text {aut }}(B, t r)$ :

$$
\mathbf{C}^{*}<\left(u_{i j}\right)_{i, j=1, \ldots, n} \mid u=\left(u_{i j}\right) \text { is unitary, } \eta \in \operatorname{Hom}(1, u), \mu \in \operatorname{Hom}\left(u^{\otimes 2}, u\right)>
$$

(i) There exists a unique structure of finitely generated full Woronowicz-Kac algebra on $A^{\text {aut }}(B, t r)$ which makes $u$ a corepresentation. There exists a unique coaction $\beta$ of $A^{\text {aut }}(B, t r)$ on $(B, t r)$ such that $u_{\beta}=u$ via the above identification $B \simeq \mathbf{C}^{n}$.

(ii) If $A$ is a finitely generated full Woronowicz-Kac algebra and $\gamma$ is a coaction of $A$ on $(B, t r)$ then there exists a unique morphism $f: A^{\text {aut }}(B, t r) \rightarrow A$ such that $(i d \otimes f) \beta=\gamma$. Moreover, $\left(A^{\text {aut }}(B, t r), \beta\right)$ is the unique pair (finitely generated full Woronowicz-Kac algebra, coaction of it on B) having this property.

The definition of $A^{\text {aut }}(B, t r)$ should be understood as follows. Let $F$ be the free *-algebra on $n^{2}$ variables $\left(u_{i j}\right)_{i, j=1, \ldots, n}$ and let $u=\left(u_{i j}\right) \in \mathcal{L}\left(\mathbf{C}^{n}\right) \otimes F$. By explicitating the notations for Hom and $\otimes$ with $D=F$ we see that both conditions $\eta \in \operatorname{Hom}(1, u)$ and $\mu \in \operatorname{Hom}\left(u^{\otimes 2}, u\right)$, as well as the condition " $u$ is unitary", could be interpreted as being a collection of relations between the $u_{i j}$ 's and their adjoints. Let $J \subset F$ be the two-sided $*$-ideal generated by all these relations. Then the matrix $u=\left(u_{i j}\right)$ is unitary in $M_{n}(\mathbf{C}) \otimes(F / J)$, so its coefficients $u_{i j}$ are of norm $\leq 1$ for every $\mathbf{C}^{*}$-seminorm on $F / J$ and the enveloping $\mathbf{C}^{*}$-algebra of $F / J$ is well-defined. We call it $A^{\text {aut }}(B, t r)$. The discussion on the (in)dependence of $A^{\text {aut }}(B, t r)$ on the basis of $B$ is left to the reader. 
We will use freely the terminology from [13] concerning concrete monoidal $\mathbf{W}^{*}$ categories. We recall that the word "concrete" comes from the fact that the monoidal $\mathbf{W}^{*}$-category is given together with an embedding into (= faithful monoidal $\mathbf{W}^{*}$-functor to) the category of finite dimensional Hilbert spaces.

Proposition 1.1. The concrete monoidal $\mathbf{W}^{*}$-category $\operatorname{Corep}\left(A^{\text {aut }}(B, t r)\right)$ of finite dimensional unitary smooth corepresentations of $A^{\text {aut }}(B, t r)$ is the completion in the sense of [13] of the concrete monoidal $\mathbf{W}^{*}$-category $\mathcal{C}_{B, t r}$ defined as follows:

- the monoid of objects of $\mathcal{C}_{B, t r}$ is $(\mathbf{N},+)$.

- the Hilbert space associated to an object $m \in \mathbf{N}$ is $B^{\otimes m}$.

- $\mathcal{C}_{B, t r}$ is the smallest concrete monoidal $\mathbf{W}^{*}$-category containing the arrows $\eta, \mu$.

The definition of $\mathcal{C}_{B, t r}$ should be understood as follows: its arrows are linear combinations of (composable) compositions of tensor products of maps of the form $\eta, \mu, \eta^{*}, \mu^{*}$ and $i d_{m}:=$ identity of $B^{\otimes m}$. It is clear that $\mathcal{C}_{B, t r}$ is a concrete monoidal $\mathbf{W}^{*}$-category.

Proof. We will prove both results at the same time. Let us consider the concrete monoidal $\mathbf{W}^{*}$-category $\mathcal{C}_{B, t r}$ in proposition 1.1 . It is clear that the pair $\left(A^{\text {aut }}(B, t r), u\right)$ in theorem 1.1 is its universal admissible pair in the sense of [13.

We prove now that the object 1 of $\mathcal{C}_{B, t r}$ is a complex conjugation for itself in the sense of [13]. Let us define an invertible antilinear map $j: B \rightarrow B$ by $j\left(b_{q}\right)=b_{q}^{*}$ for any $q$. With the notations from page 39 in 13 we have

$$
t_{j}(1)=\sum_{p} b_{p} \otimes j\left(b_{p}\right)=\sum_{p} b_{p} \otimes b_{p}^{*}=\sum_{p q} \operatorname{tr}\left(b_{p}^{*} b_{q}^{*}\right) b_{p} \otimes b_{q}
$$

We have seen in proof of lemma 1.2 that $\mu^{*} \eta(1)$ is given by the same formula, and it follows that $t_{j}=\mu^{*} \eta$. In particular we get that $t_{j} \in \operatorname{Hom}_{\mathcal{C}_{B, t r}}(0,2)$. By choosing as basis $X=\left\{b_{1}, b_{2}, \ldots, b_{n}\right\}$ of $B$ a complete system of matrix units we may assume that $X=X^{*}$. It follows that $j=j^{-1}$, so with the notations in [13] we get that $\bar{t}_{j}=t_{j-1}^{*}=t_{j}^{*}$ is in $\operatorname{Hom}_{\mathcal{C}_{B, t r}}(2,0)$. Thus $1=\overline{1}$ in $\mathcal{C}_{B, t r}$ in the sense of [13. Notice that this shows also that $1=\overline{1}$ in $\mathcal{C}_{B, t r}$ in the sense of [7]. Moreover, from

$$
\left\|t_{j}(1)\right\|^{2}=\sum_{p q}<b_{p} \otimes b_{p}^{*}, b_{q} \otimes b_{q}^{*}>=n
$$

we get that the dimension $d_{\mathcal{C}_{B, t r}}(1)$ of 1 in $\mathcal{C}_{B, t r}$ in the sense of [7] is equal to $n$.

As $1=\overline{1}$ theorem 1.3 in [13] applies and shows that $\left(A^{\text {aut }}(B, t r), u\right)$ is a finitely generated Woronowicz algebra (i.e. a compact matrix pseudogroup, with the terminology in there) whose concrete monoidal $\mathbf{W}^{*}$-category of corepresentations is the completion of $\mathcal{C}_{B, t r}$. Also $A^{\text {aut }}(B, t r)$ is full by definition, and it is of Kac type because the quantum dimension of its fundamental corepresentation $u$ is $d_{\mathcal{C}_{B, t r}}(1)=n$, hence is equal to its classical dimension (see [7], see also section 1 in [2]).

Summing up, we have proved both the first assertion in theorem 1.1 (i) and proposition 1.1. The other assertions in theorem 1.1 follow from lemmas 1.1 and 1.2. 


\section{GOOD TRACE, GOOD INDEX}

Let $B$ be a finite dimensional $\mathbf{C}^{*}$-algebra. Let $n=\operatorname{dim}(B)$. Denote by $i d_{s}$ the identity of $\mathcal{L}\left(B^{\otimes s}\right)$ for any $s$. As in section 1 , we denote by $\mu: B \otimes B \rightarrow B$ the multiplication and by $\eta: \mathbf{C} \rightarrow B$ the linear unital map.

We recall that each faithful trace on $B$ makes it into a Hilbert space, so in particular it gives rise to adjoints $\mu^{*}: B \rightarrow B \otimes B$ and $\eta^{*}: B \rightarrow \mathbf{C}$.

Proposition 2.1. If $t r: B \rightarrow \mathbf{C}$ is a faithful normalised trace then the following are equivalent:

(i) $\operatorname{tr}$ is the restriction of the unique trace of $\mathcal{L}(B)$, via the embedding $B \subset \mathcal{L}(B)$ given by the left regular representation.

(ii) $\mathbf{C} \subset B$ is a Markov inclusion in the sense of [5].

(iii) if $\phi: B \simeq \bigoplus_{\gamma=1}^{s} M_{m_{\gamma}}$ is a decomposition of $B$ as a multimatrix algebra, then the weights $\lambda_{\gamma}:=\operatorname{tr}\left(\phi^{-1}\left(I_{M_{m_{\gamma}}}\right)\right)$ of tr are given by $\lambda_{\gamma}=n^{-1} m_{\gamma}^{2}$ for any $\gamma$.

(iv) $\mu \mu^{*}=n \cdot i d$.

Proof. The equivalence between (i), (ii) and (iii) is clear from definitions. Let us prove that (iii) and (iv) are equivalent. We may identify $B$ with a multimatrix algebra $\bigoplus_{\gamma=1}^{s} M_{m_{\gamma}}$ as in (iii). Let $\left\{\lambda_{\gamma}\right\}$ be the weights of $\operatorname{tr}$. Then $\operatorname{tr}\left(e_{i j}^{\gamma}\right)=\delta_{i, j} m_{\gamma}^{-1} \lambda_{\gamma}$ for any $\gamma, i, j$, so the set

$$
\left\{f_{i j}^{\gamma}:=m_{\gamma}^{1 / 2} \lambda_{\gamma}^{-1 / 2} e_{i j}^{\gamma} \mid \gamma=1,2, \ldots, s, i, j=1,2, \ldots, m_{\gamma}\right\}
$$

is an orthonormal basis of $B$. Thus for any $\gamma, i, j$ we have

$$
\begin{gathered}
\mu^{*}\left(e_{i j}^{\gamma}\right)=\sum_{\delta \varepsilon k l p q} f_{k l}^{\delta} \otimes f_{p q}^{\varepsilon}<\mu^{*}\left(e_{i j}^{\gamma}\right), f_{k l}^{\delta} \otimes f_{p q}^{\varepsilon}>=\sum_{\delta \varepsilon k l p q} f_{k l}^{\delta} \otimes f_{p q}^{\varepsilon} \operatorname{tr}\left(f_{k l}^{\delta} f_{p q}^{\varepsilon} e_{j i}^{\gamma}\right)= \\
\sum_{\delta \varepsilon k l p q} m_{\delta}^{1 / 2} \lambda_{\delta}^{-1 / 2} e_{k l}^{\delta} \otimes m_{\varepsilon}^{1 / 2} \lambda_{\varepsilon}^{-1 / 2} e_{p q}^{\varepsilon} \operatorname{tr}\left(m_{\delta}^{1 / 2} \lambda_{\delta}^{-1 / 2} e_{k l}^{\delta} m_{\varepsilon}^{1 / 2} \lambda_{\varepsilon}^{-1 / 2} e_{p q}^{\varepsilon} e_{j i}^{\gamma}\right)=m_{\gamma} \lambda_{\gamma}^{-1} \sum_{l} e_{i l}^{\gamma} \otimes e_{l j}^{\gamma}
\end{gathered}
$$

Thus for any $\gamma, i, j$ we have $\mu \mu^{*}\left(e_{i j}^{\gamma}\right)=m_{\gamma} \lambda_{\gamma}^{-1} \sum_{l} e_{i j}^{\gamma}=m_{\gamma}^{2} \lambda_{\gamma}^{-1} e_{i j}^{\gamma}$, so (iii) $\Longleftrightarrow$ (iv).

Definition 2.1. The distinguished trace in proposition 2.1, say $\tau$, will be called the canonical trace of $B$. The finitely generated full Woronowicz-Kac algebra $A^{\text {aut }}(B, \tau)$ in theorem 1.1 will be denoted $A^{\text {aut }}(B)$. The concrete monoidal $\mathbf{W}^{*}$-category $\mathcal{C}_{B, \tau}$ in proposition 1.1 wil be denoted $\mathcal{C}_{B}$.

The arrows $\eta, \mu, \eta^{*}, \mu^{*}$ could be thought of as being "generators" of $\mathcal{C}_{B}$. In the next lemma we collect the relevant "relations" satisfied by these arrows.

Lemma 2.1. (i) $\mu \mu^{*}=n \cdot i d$ and $\eta^{*} \eta=i d$.

(ii) $(\mu \otimes i d)\left(i d \otimes \mu^{*}\right)=(i d \otimes \mu)\left(\mu^{*} \otimes i d\right)=\mu^{*} \mu$.

(iii) $\mu(\mu \otimes i d)=\mu(i d \otimes \mu)$.

(iv) $\mu(i d \otimes \eta)=\mu(\eta \otimes i d)=i d$.

Proof. We have $\eta^{*} \eta(1)=<\eta^{*} \eta(1), 1>=<\eta(1), \eta(1)>=<1,1>=\operatorname{tr}(1)=1$. Also the equality $\mu \mu^{*}=i d$ was already proved, and (iii) and (iv) are trivial, so it remains to 
prove (ii). For, we may use an identification $B=\bigoplus_{\gamma=1}^{s} M_{m_{\gamma}}$ as in proof of proposition 2.1. By using the formula of $\mu^{*}$ in there (with $\lambda_{\gamma}=n^{-1} m_{\gamma}^{2}$ !) we get

$$
\begin{gathered}
(\mu \otimes i d)\left(i d \otimes \mu^{*}\right)\left(e_{p q}^{\delta} \otimes e_{i j}^{\gamma}\right)=n m_{\gamma}^{-1} \sum_{l}(\mu \otimes i d)\left(e_{p q}^{\delta} \otimes e_{i l}^{\gamma} \otimes e_{l j}^{\gamma}\right)=n m_{\gamma}^{-1} \delta_{\gamma, \delta} \delta_{q, i} \sum_{l} e_{p l}^{\gamma} \otimes e_{l j}^{\gamma} \\
(i d \otimes \mu)\left(\mu^{*} \otimes i d\right)\left(e_{p q}^{\delta} \otimes e_{i j}^{\gamma}\right)=n m_{\delta}^{-1} \sum_{l}(i d \otimes \mu)\left(e_{p l}^{\delta} \otimes e_{l q}^{\delta} \otimes e_{i j}^{\gamma}\right)=n m_{\delta}^{-1} \delta_{\gamma, \delta} \delta_{q, i} \sum_{l} e_{p l}^{\gamma} \otimes e_{l j}^{\gamma} \\
\mu^{*} \mu\left(e_{p q}^{\delta} \otimes e_{i j}^{\gamma}\right)=\delta_{\gamma, \delta} \delta_{q, i} \mu^{*}\left(e_{p j}^{\gamma}\right)=\delta_{\gamma, \delta} \delta_{q, i} n m_{\gamma}^{-1} \sum_{l} e_{p l}^{\gamma} \otimes e_{l j}^{\gamma}
\end{gathered}
$$

for any $\gamma, \delta, i, j, p, q$, and this proves (ii).

We recall that for $m \in \mathbf{N}$ and $\beta>0$ the $m$-th Temperley-Lieb algebra $A_{\beta, m}$ of index $\beta$ is defined with generators $e_{1}, e_{2}, \ldots, e_{m-1}$ and Jones' relations (see [6]):

$e_{i}=e_{i}^{*}=e_{i}^{2}$ for any $i$.

$e_{i} e_{j}=e_{j} e_{i}$ for any $i$ and $j$ with $|i-j| \geq 2$.

$\beta e_{i} e_{j} e_{i}=e_{i}$ for $i$ and $j$ with $|i-j|=1$.

Proposition 2.2. Assume that $n \geq 4$ and let $k \in \mathbf{N}$.

(i) $P=n^{-1} \mu^{*} \mu \in \mathcal{L}(B \otimes B)$ and $Q=\eta \eta^{*} \in \mathcal{L}(B)$ are projections satisfying

$$
\begin{gathered}
n(Q \otimes i d) P(Q \otimes i d)=(Q \otimes i d) \\
n(i d \otimes Q) P(i d \otimes Q)=(i d \otimes Q) \\
n P(i d \otimes Q) P=n P(Q \otimes i d) P=P \\
(P \otimes i d)(i d \otimes P)=(i d \otimes P)(P \otimes i d)
\end{gathered}
$$

(ii) There exists a faithful representation $\pi_{k}: A_{n, 2 k} \rightarrow \mathcal{L}\left(B^{\otimes k}\right)$ which sends

$$
\begin{gathered}
e_{2 s} \mapsto i d_{s-1} \otimes P \otimes i d_{k-s-1} \quad(s=1,2, \ldots, k-1) \\
e_{2 s+1} \mapsto i d_{s} \otimes Q \otimes i d_{k-s-1} \quad(s=0,1, \ldots, k-1)
\end{gathered}
$$

(iii) $\operatorname{dim}\left(\operatorname{End}_{\mathcal{C}_{B}}(k)\right) \geq C_{2 k}$ (the $2 k$-th Catalan number).

Proof. The assertions in (i) follow via easy computations from the formulas in lemma 2.1. From (i) we get Jones' relations, hence a representation $\pi_{k}$ as in (ii). This representation is nothing but the well-known one coming by applying basic constructions to the Markov inclusion $\mathbf{C} \subset B$ (cf. proposition 2.1 (ii)), so it is faithful. As $\mu$ and $\eta$ are arrows of $\mathcal{C}_{B}$, it follows that $P \in \operatorname{End}_{\mathcal{C}_{B}}(2)$ and $Q \in \operatorname{End}_{\mathcal{C}_{B}}(1)$. Thus (ii) gives a copy of $A_{n, 2 k}$ into $\operatorname{End}_{\mathcal{C}_{B}}(k)$ for any $k$. On the other hand, as the index $n \geq 4$ is generic, we have by [6] that $\operatorname{dim}\left(A_{n, 2 k}\right)=C_{2 k}$, and this proves (iii).

We will see in next section that $\operatorname{End}_{\mathcal{C}_{B}}(k) \simeq A_{n, 2 k}$ for any $k$. 


\section{Computation of $\operatorname{Hom}_{\mathcal{C}_{B}}(0, k)$}

We fix an algebra $B$ of dimension $n \geq 4$ and we use the notations in section 2 .

Lemma 3.1. Let $\mathcal{C}_{B}{ }^{+}$be the set of arrows of $\mathcal{C}_{B}$ consisting of linear combinations of compositions of tensor products of maps of the form $\eta, \mu^{*}$ and $i d_{1}$. Then each arrow of $\mathcal{C}_{B}$ is a linear combination of compositions of the form ab $b^{*}$, with $a, b$ arrows in $\mathcal{C}_{B}{ }^{+}$.

Proof. By definitions the arrows of $\mathcal{C}_{B}$ are linear combinations of compositions of tensor products of maps of the form $\eta, \mu^{*}, \eta^{*}, \mu$ and $i d_{1}$. By an easy induction argument, it suffices to prove is that each composition of the form $\left(i d_{\text {? }} \otimes x \otimes i d_{\text {? }}\right)\left(i d_{\text {? }} \otimes y \otimes i d_{\text {? }}\right)$ with $x \in\left\{\eta^{*}, \mu, i d\right\}$ and $y \in\left\{\eta, \mu^{*}, i d\right\}$ may be written as $\lambda\left(i d_{?} \otimes z \otimes i d_{?}\right)\left(i d_{?} \otimes t \otimes i d_{?}\right)$ with $z \in\left\{\eta, \mu^{*}, i d\right\}$ and $t \in\left\{\eta^{*}, \mu, i d\right\}$ and $\lambda \in \mathbf{C}$ (i.e. that "modulo scalars, in each composition giving rise to an arrow of $\mathcal{C}_{B}$, the $\eta^{*}$ 's and $\mu$ 's can be moved to the right"). All $3 \times 3=9$ assertions to be verified are clear from lemma 2.1.

Lemma 3.2. For any $p \geq 0$ define $\left(\mu^{*}\right)^{(p)} \in \operatorname{Hom}_{\mathcal{C}_{B}}(1, p+1)$ by $\left(\mu^{*}\right)^{(0)}=i d_{1}$ and by

$$
\left(\mu^{*}\right)^{(p)}=\left(i d_{p-1} \otimes \mu^{*}\right)\left(i d_{p-2} \otimes \mu^{*}\right) \ldots\left(i d \otimes \mu^{*}\right) \mu^{*}
$$

if $p \geq 1$. Define also for any $p \geq 1$ an arrow $\eta^{(p)} \in \operatorname{Hom}_{\mathcal{C}_{B}}(0, p)$ by $\eta^{(p)}=\left(\mu^{*}\right)^{(p-1)} \eta$. Then for any $k \in \mathbf{N}$ each arrow in $\operatorname{Hom}_{\mathcal{C}_{B}}(0, k)$ is a linear combination of compositions of maps of the form $i d_{a} \otimes \eta^{(p)} \otimes i d_{b}$, with $a, b, p \in \mathbf{N}$.

Proof. We know from lemma 3.1 that each arrow in $\operatorname{Hom}_{\mathcal{C}_{B}}(0, k)$ is a linear combination of arrows in $\mathcal{C}_{B}{ }^{+}$. In particular each arrow in $\operatorname{Hom}_{\mathcal{C}_{B}}(0, k)$ is a linear combination of compositions of arrows of the form $i d_{?} \otimes \eta^{(?)} \otimes i d_{?}$ and $i d_{?} \otimes \mu^{*} \otimes i d_{?}$. It is enough to prove that each such composition may be written without $\mu^{*}$ 's. For, let us choose such a composition, say $C$, having $z \geq 1 \mu^{*}$ s in its writing, and assume that $C$ is not equal to a composition having $z-1 \mu^{*}$ 's in its writing. Then $C$ is of the form $C^{\prime}\left(i d_{a} \otimes \mu^{*} \otimes i d_{b}\right) D$ where $C^{\prime}$ has $z-1 \mu^{*}$ s in its writing, $a, b \in \mathbf{N}$, and $D$ is a composition of arrows of the form $i d_{\text {? }} \otimes \eta^{(?)} \otimes i d_{\text {? }}$. By using an easy minimality argument on the lenght of $D$ ("the term containing $\mu^{*}$ cannot be moved to the right") we may assume that $D$ is of the form $\left(i d_{c} \otimes \eta^{(p)} \otimes i d_{d}\right) D^{\prime}$ with $a+1 \in\{c+1, c+2, \ldots, c+p\}$, i.e. that

$$
C=C^{\prime}\left(i d_{c} \otimes\left(\left(i d_{a-c} \otimes \mu^{*} \otimes i d_{b-d}\right)\left(\mu^{*}\right)^{(p)} \eta\right) \otimes i d_{d}\right) D^{\prime}
$$

From the coassociativity property $\left(\mu^{*} \otimes i d\right) \mu^{*}=\left(i d \otimes \mu^{*}\right) \mu^{*}$ of $\mu^{*}$ (cf. lemma 2.1 (iii)) we get that the term in the middle is $\left(\mu^{*}\right)^{(p+1)} \eta=\eta^{(p+1)}$, contradiction.

Lemma 3.3. Define a set $X_{k} \subset \operatorname{Hom}_{\mathcal{C}_{B}}(0, k)$ for any $k \geq 1$ in the following way. $X_{1}=\{\eta\}$ and for any $k \geq 2$

$$
X_{k}=\left\{\left(i d_{x} \otimes \alpha \otimes i d_{y} \otimes \beta \otimes i d_{z} \otimes \gamma \otimes \ldots\right) \eta^{(p)}\right\}
$$

with $p$ ranging over $\{1,2, \ldots, k\}, x, y, z, \ldots$ ranging over sequences of strictly positive integers whose sum is $p$, and $\alpha \in X_{a}, \beta \in X_{b}, \gamma \in X_{c}$ etc., with $a, b, c, \ldots$ being positive integers whose sum is $k-p$.

Then $X_{k}$ is a system of generators of $\operatorname{Hom}_{\mathcal{C}_{B}}(0, k)$ for any $k$. 
Proof. We know from lemma 3.2 that for any $k$ the set $Y_{k}$ of compositions of maps of the form $i d_{a} \otimes \eta^{(p)} \otimes i d_{b}$ with $a, b, p \in \mathbf{N}$ which happen to belong to $H_{o m_{\mathcal{C}_{B}}}(0, k)$ is a system of generators of $\operatorname{Hom}_{\mathcal{C}_{B}}(0, k)$. We will prove by induction on $k$ that $X_{k}=Y_{k}$. For $k=1$ this is clear from lemma 3.2, so let $k \geq 2$. Pick an arbitrary element of $Y_{k}$

$$
C=\circ_{i=1}^{i=s}\left(i d_{a_{i}} \otimes \eta^{\left(p_{i}\right)} \otimes i d_{b_{i}}\right)
$$

We have $a_{s}=b_{s}=0$. It's easy to see that imposing the condition that the biggest integer $t$ such that $a_{t}=0$ is minimal is the same as assuming that $a_{1}, a_{2}, \ldots, a_{s-1}$ are strictly positive numbers. With $x:=\inf \left\{a_{1}, a_{2}, \ldots, a_{s-1}\right\}$ and $p:=p_{s}$ we get that

$$
C=\left(i d_{x} \otimes f\right) \eta^{(p)}
$$

with $f$ being a composition of maps of the form $i d_{a} \otimes \eta^{(q)} \otimes i d_{b}$ which belongs to $H_{o m_{\mathcal{C}_{B}}}(p, k-x)$, i.e. with $f$ being of the form $\alpha \otimes i d_{y} \otimes \beta \otimes i d_{z} \otimes \gamma \otimes \ldots$ with $x, y, z, \ldots, \alpha, \beta, \gamma, \ldots$ and $a, b, c, \ldots$ being as in the definition of $X_{k}$ 's.

Lemma 3.4. $\operatorname{dim}\left(\operatorname{Hom}_{\mathcal{C}_{B}}(0, k)\right) \leq C_{k}:=\frac{(2 k) !}{k !(k+1) !}$ (the $k$-th Catalan number) $\forall k$.

Proof. With $D_{k}=\operatorname{dim}\left(\operatorname{Hom}_{\mathcal{C}_{B}}(0, k)\right)$ lemma 3.3 shows that

$$
D_{k} \leq D_{k-1}+\sum_{a+b=k-2} D_{a} D_{b}+\sum_{a+b+c=k-2} D_{a} D_{b} D_{c}+\cdots
$$

(here each sum corresponds to a value of $p$ in lemma 3.3, and $a, b, c, \ldots$ are the ones in lemma 3.3). Thus $D_{k} \leq E_{k}$, where $E_{k}$ are the numbers defined by $E_{0}=E_{1}=1$ and by the above formula with $E_{m}$ at the place of $D_{m}$ and with $=$ at the place of $\leq$. By rearranging terms in this equality we get (by an easy induction on $s$ ) that

$$
E_{s}=\sum_{x+y=s-1} E_{x} E_{y}
$$

for any $s$. It follows that the $E_{s}$ 's are the Catalan numbers (well-known, just consider the square of the series $\left.\sum E_{s} z^{s} \ldots\right)$ and we are done.

Proposition 3.1. Let $k \in \mathbf{N}$.

(i) The inclusion $A_{n, 2 k} \subset \operatorname{End}_{\mathcal{C}_{B}}(k)$ given by proposition 2.2 is an equality.

(ii) The set $X_{k}$ in lemma 3.3 is a basis of $\operatorname{Hom}_{\mathcal{C}_{B}}(0, k)$.

(iii) $\operatorname{dim}\left(\operatorname{Hom}_{\mathcal{C}_{B}}(0, k)\right)=C_{k}$.

Proof. For any $l \in \mathbf{N}$ we have

$$
\operatorname{dim}\left(\operatorname{End}_{\mathcal{C}_{B}}(l)\right)=\operatorname{dim}\left(\operatorname{Hom}_{\mathcal{C}_{B}}(0,2 l)\right) \leq C_{2 l} \leq \operatorname{dim}\left(\operatorname{End}_{\mathcal{C}_{B}}(l)\right)
$$

Indeed, by Frobenius reciprocity we have $\operatorname{End}_{\mathcal{C}_{B}}(l) \simeq \operatorname{Hom}_{\mathcal{C}_{B}}(0,2 l)$ for any $l$, and this gives the equality on the left. Lemma 3.4 and proposition 2.2 give the inequalities.

Thus both inequalities have to be equalities, and this proves (i). This proves also (ii,iii) for even values of $k$. By definition of $X_{k}$ we have $X_{k} \otimes \eta \subset X_{k+1}$, and with $k=2 l+1$ we get that the set $X_{2 l+1} \otimes \eta$ consists of linearly independent maps. Thus $X_{2 l+1}$ consists of linearly independent maps, so we get (ii,iii) for odd values of $k$. 


\section{Corepresentations of $A^{\text {aut }}(B)$. Applications}

Let $B$ be a finite dimensional $\mathbf{C}^{*}$-algebra of dimension $\geq 4$.

Theorem 4.1. The set of classes of finite dimensional irreducible smooth corepresentations of $A^{\text {aut }}(B)$ can be labeled by the positive integers, $\operatorname{Irr}\left(A^{\text {aut }}(B)\right)=\left\{p_{n} \mid n \in \mathbf{N}\right\}$, such that the fusion rules are

$$
p_{k} \otimes p_{s}=p_{|k-s|}+p_{|k-s|+1}+\cdots+p_{k+s-1}+p_{k+s}
$$

Proof. We will use proposition 3.1 (iii) and a method from [1].

We first recall a few well-known facts on $\mathbf{S O}(3)$. Let $\chi_{0}, \chi_{1}, \chi_{2}, \chi_{3}, \ldots$ be the characters of the irreducible representations of $\mathbf{S O}(3)$, listed in the increasing order of dimensions. They satisfy the formulas in theorem 4.1, with $\chi_{m}$ at the place of $p_{m}$ and with $\cdot$ at the place of $\otimes$. We denote by $C(\mathbf{S O}(3))_{c}$ the $*$-subalgebra of $C(\mathbf{S O}(3))$ generated by the $\chi_{i}$ 's. Let $\int$ be the integration over $\mathbf{S O}(3)$. Then the $\chi_{i}$ 's form an orthonormal basis of $\left(C(\mathbf{S O}(3))_{c}, \int\right)$. There exists a canonical isomorphism

$$
\mathbf{C}[X] \simeq C(\mathbf{S O}(3))_{c}, \quad X \mapsto \chi_{1}
$$

For any $k$ the multiplicity of 1 into $\left(1+\chi_{1}\right)^{k}$ is $C_{k}$. See e.g. $\mathbb{1}$ with $F=\left(\begin{array}{cc}0 & 1 \\ -1 & 0\end{array}\right)$ for everything (we didn't succeed in finding a classical reference).

Let $A^{\text {aut }}(B)_{c}$ be the algebra of characters of corepresentations of $A^{\text {aut }}(B)$, let $h$ : $A^{\text {aut }}(B) \rightarrow \mathbf{C}$ be the Haar functional, and denote by $r \rightarrow \chi(r)$ the character of corepresentations (see [12]). By using the above isomorphism, we may define a morphism of algebras in the following way.

$$
\phi: C(\mathbf{S O}(3))_{c} \rightarrow A^{a u t}(B)_{c}, \quad \chi_{1} \mapsto \chi(u)-1
$$

We have on one hand that the multiplicity of 1 into $\left(1+\chi_{1}\right)^{k}$ is $C_{k}$, and on the other hand that the multiplicity of 1 into $u^{\otimes k}$ is also $C_{k}$ (cf. proposition 3.1 (iii) and the definition of $\mathcal{C}_{B}$ ). This could be interpreted as saying that

$$
h \phi\left(\left(\chi_{1}+1\right)^{k}\right)=\int\left(\chi_{1}+1\right)^{k}
$$

for any $k$. It's easy to get from this (by induction on $s$ ) that $h \phi\left(\chi_{1}^{s}\right)=\int\left(\chi_{1}^{s}\right)$ for any $s$, and as $\chi_{1}$ generates $C(\mathbf{S O}(3))_{c}$ as an algebra we get that

$$
h \phi=\int
$$

Thus $\left\{\phi\left(\chi_{k}\right) \mid k \geq 0\right\}$ is an orthonormal basis of $A^{\text {aut }}(B)_{c}$, and for finishing the proof it's enough to construct corepresentations $p_{k}$ of $A^{\text {aut }}(B)$ such that $\chi\left(p_{k}\right)=\phi\left(\chi_{k}\right)$ for any $k$. Indeed, the fact that $\operatorname{Irr}\left(A^{\text {aut }}(B)\right)=\left\{p_{n} \mid n \in \mathbf{N}\right\}$ will be clear from this and from Peter-Weyl type theory from [12]; and the assertion on fusion rules will be also clear from this and from the fusion rules for irreducible representations of $\mathbf{S O}(3)$.

We do it by induction on $k$. We may define $p_{0}=1$. Also as $\eta \in \operatorname{Hom}(1, u)$ we know that $u$ contains a copy of 1 , so we may set $p_{1}=u-1$. So let $k \geq 2$ and assume that we have constructed $p_{0}, p_{1}, \ldots, p_{k-1}$ with

$$
\chi\left(p_{i}\right)=\phi\left(\chi_{i}\right), \quad i=0,1, \ldots, k-1
$$


We know from fusion rules for representations of $\mathbf{S O}(3)$ that

$$
\chi_{k-1} \chi_{1}=\chi_{k-2}+\chi_{k-1}+\chi_{k}
$$

so by applying $\phi$ and by using $h \phi=\int$ we get that both $p_{k-2}$ and $p_{k-1}$ are subcorepresentations of $p_{k-1} \otimes p_{1}$. Thus there exists a corepresentation $p_{k}$ such that

$$
p_{k-1} \otimes p_{1}=p_{k-2}+p_{k-1}+p_{k}
$$

From the above two formulas we get $\chi\left(p_{k}\right)=\phi\left(\chi_{k}\right)$ and we are done.

We recall from Wang [8] that for $m \geq 2$ the $\mathbf{C}^{*}$-algebra $A_{o}(m)$ is defined with selfadjoint generators $\left\{v_{i j}\right\}_{i, j=1, \ldots, m}$ and the relations making the matrix $v=\left(v_{i j}\right)$ unitary. The pair $\left(A_{o}(m), v\right)$ is a finitely generated full Woronowicz-Kac algebra.

Corollary 4.1. For any $m \geq 2$ the enveloping $\mathbf{C}^{*}$-algebra of the $*$-subalgebra of $A_{o}(m)$ generated by the coefficients of the square of its fundamental corepresentation is canonically isomorphic (as a full Woronowicz-Kac algebra) to $A^{\text {aut }}\left(M_{m}(\mathbf{C})\right.$ ).

Proof. Let $\mathcal{X}=*-\operatorname{alg}\left(\left\{v_{i j} v_{k l}\right\}_{i, j, k, l=1, \ldots, m}\right)$ be the $*$-algebra in the statement and let $X$ be its enveloping $\mathbf{C}^{*}$-algebra. Then the pair $\left(X, v^{\otimes 2}\right)$ is a finitely generated full Woronowicz-Kac algebra. We have the following two facts.

I. We recall that the fusion semiring $R^{+}(A)$ of a Woronowicz-Kac algebra $A$ is the set of equivalence classes of corepresentations of $A$ together with the operations sum and tensor product. By [1] we have an isomorphism

$$
R^{+}\left(A_{o}(m)\right) \simeq R^{+}(C(\mathbf{S U}(2)))
$$

sending $v$ onto the fundamental corepresentation of $C(\mathbf{S U}(2))$. It follows that we have an isomorphism $R^{+}(X) \simeq R^{+}(C(\mathbf{S O}(3)))$ sending $v^{\otimes 2}$ onto the sum of the fundamental corepresentation of $C(\mathbf{S O}(3))$ with the trivial corepresentation. On the other hand, theorem 4.1 gives an isomorphism

$$
R^{+}\left(A^{\text {aut }}\left(M_{m}(\mathbf{C})\right)\right) \simeq R^{+}(C(\mathbf{S O}(3)))
$$

which sends $u$ onto the sum of the fundamental corepresentation of $C(\mathbf{S O}(3))$ with the trivial corepresentation. By combining these two facts we get an isomorphism

$$
g: R^{+}\left(A^{\text {aut }}\left(M_{m}(\mathbf{C})\right)\right) \simeq R^{+}(X)
$$

which sends $u \mapsto v^{\otimes 2}$.

II. Consider the fundamental coaction $\phi$ of $A_{o}(m)$ on $M_{m}(\mathbf{C})$, i.e. the map $\phi$ : $M_{m}(\mathbf{C}) \rightarrow M_{m}(\mathbf{C}) \otimes A_{o}(m)$ given by $x \mapsto a d(v)(x \otimes 1)$. The image of $\phi$ is contained in $M_{m}(\mathbf{C}) \otimes \mathcal{X}$, so we get by restriction a coaction $\psi: M_{m}(\mathbf{C}) \rightarrow M_{m}(\mathbf{C}) \otimes X$. It follows that there exists a morphism of $\mathbf{C}^{*}$-algebras

$$
f: A^{\text {aut }}\left(M_{m}(\mathbf{C})\right) \rightarrow X
$$

such that $(i d \otimes f) \beta=\psi$, where $\beta$ is the fundamental coaction of $A^{\text {aut }}\left(M_{m}(\mathbf{C})\right)$ on $M_{m}(\mathbf{C})$. Thus at the level of corepresentations we get a morphism of semirings

$$
f_{*}: R^{+}\left(A^{\text {aut }}\left(M_{m}(\mathbf{C})\right)\right) \rightarrow R^{+}(X)
$$

which sends $u \mapsto v^{\otimes 2}$.

By using I and II we get that $g^{-1} f_{*}$ is an endomorphism of $R^{+}\left(A^{\text {aut }}\left(M_{m}(\mathbf{C})\right)\right)$ sending $u \mapsto u$. By using the structure of $R^{+}\left(A^{\text {aut }}\left(M_{m}(\mathbf{C})\right)\right)$ given by the fusion rules 
in theorem 4.1 we get from this that $g^{-1} f_{*}=i d$, so in particular $f_{*}$ is an isomorphism. It follows that $f$ maps any basis of $\mathcal{A}^{\text {aut }}\left(M_{m}(\mathbf{C})\right)$ consisting of coefficients of irreducible corepresentations (cf. Woronowicz' Peter-Weyl theory, see [12]) onto a basis of $\mathcal{X}$ consisting of coefficients of irreducible corepresentations. Thus the restriction $\mathcal{A}^{\text {aut }}\left(M_{m}(\mathbf{C})\right) \rightarrow \mathcal{X}$ of $f$ is an isomorphism, and by passing at the enveloping $\mathrm{C}^{*}$-algebras level we get that $f$ itself is an isomorphism.

Corollary 4.2. $A^{\text {aut }}(B)$ is amenable as a Woronowicz algebra iff $\operatorname{dim}(B)=4$.

Proof. Theorem 4.1 gives an isomorphism

$$
R^{+}\left(A^{\text {aut }}(B)\right) \simeq R^{+}(C(\mathbf{S O}(3)))
$$

which sends the fundamental $\operatorname{dim}(B)$-dimensional corepresentation of $A^{\text {aut }}(B)$ onto a 4-dimensional corepresentation of $C(\mathbf{S O}(3))$ (see the proof of corollary 4.1). We may conclude by applying the following consequence of the quantum Kesten result (proposition 6.1 in [2]): is $A, B$ are two finitely generated Woronowicz algebras, if $A$ is amenable and if $\varphi: R^{+}(A) \simeq R^{+}(B)$ is an isomorphism, then $B$ is amenable if and only if $\varphi$ is dimension-preserving.

Corollary 4.3. Let $P$ be a $I I_{1}$ factor, $\alpha$ be a minimal coaction of $A^{\text {aut }}(B)$ on $P$ and $\beta$ be the canonical coaction of $A^{\text {aut }}(B)$ on $B$. Then the fixed point subfactor (see [3])

$$
P^{\alpha} \subset\left(B^{o} \otimes P\right)^{\beta \odot \alpha}
$$

has index $\operatorname{dim}(B)$ and principal graph $A_{\infty}$.

Proof. This is clear from theorem 4.3 (ii) in [3] which asserts that the relative commutants of even order of any fixed point subfactor of the form $P^{\alpha} \subset\left(B^{o} \otimes P\right)^{\beta \odot \alpha}$ are the algebras of intertwiners of the tensor powers of the corepresentation corresponding to the coaction $\beta$. In our present situation, the corepresentation corresponding to $\beta$ is the fundamental corepresentation $u$ of $A^{\text {aut }}(B)$, the algebras of intertwiners of its tensor powers are Temperley-Lieb algebras (cf. proposition 3.1 (i); in fact this is also clear from theorem 4.1), so our subfactor has graph $A_{\infty}$.

\section{REFERENCES}

[1] T. Banica, Théorie des représentations du groupe quantique compact libre $O(n), C . R$. Acad. Sci. Paris 322 (1996), 241-244.

[2] T. Banica, Representations of compact quantum groups and subfactors, to appear in J. Reine Angew. Math., math/9804015.

[3] T. Banica, Quantum groups acting on n points, complex Hadamard matrices, and a construction of subfactors, math/9806054

[4] T. Banica, Fusion rules for representations of compact quantum groups, math/9811039.

[5] F.M. Goodman, P. de la Harpe and V.F.R. Jones, "Coxeter graphs and towers of algebras", Publ. M.S.R.I. 62, Springer (1989)

[6] V. Jones, Index for subfactors, Invent. Math. 72 (1983), 1-25.

[7] R. Longo and J. Roberts, A theory of dimension, K-Theory 11 (1997), 103-159.

[8] S. Wang, Free products of compact quantum groups, Commun. Math. Phys. 167 (1995), 671-692.

[9] S. Wang, Quantum symmetry groups of finite spaces, Commun. Math. Phys. 195 (1998), $195-211$.

[10] S. Wang, Ergodic actions of universal quantum groups on operator algebras, math/9807093.

[11] S. Wang, Structure and isomorphic classification of compact quantum groups $A_{u}(Q)$ and $B_{u}(Q)$, math/9807095. 
[12] S.L. Woronowicz, Compact matrix pseudogroups, Commun. Math. Phys. 111 (1987), 613-665.

[13] S.L. Woronowicz, Tannaka-Krein duality for compact matrix pseudogroups. Twisted $S U(n)$ groups, Invent. Math. 93 (1988), 35-76.

Institut de Mathématiques de Jussieu, Case 191, Université Paris 6, 4 Place Jussieu, 75005 PARIS

E-mail address: banica@math.jussieu.fr 